\title{
Replantation of a Multi-level Upper Extremity Amputation
}

\author{
Yefeng Yin, Xiulian Si, Jiangning Wang*, Cui Jin and Zhao Chao
}

Department of plastic and reconstructive Surgery, Beijing Luhe Hospital, Capital Medical University, Beijing 101149, China

\begin{abstract}
Multi-segmental amputation caused by machine is rare, multi-amputation injures is complex and difficult in operation and replantation. An 18-year-old boy presented with completely amputation of the right forearm, wrist and thumb. On admission, he had clear consciousness. The operation started $3 \mathrm{~h}$ after the accident and was carried out simultaneously by two replantation teams and three procedures under brachial plexus anesthesia (the right forearm team; wrist and hand team). The replanted limb, hand and thumb were survived well. Our case was unusual in replantation of severed limb. Our case stated a replantation of upper extremity with associated a very rarely seen multi-level type amputation.
\end{abstract}

Keywords: Replantation; Multiple segments; Upper extremity amputation

\section{Case Report}

In February, 2011, an 18-year-old boy presented with completely amputation of the right forearm, wrist and thumb. On admission, he had clear consciousness. His pulse was 82 , blood pressure was $180 / 96$ $\mathrm{mm} \mathrm{Hg}$. Hemoglobin concentration was $153 \mathrm{~g} / \mathrm{L}$. By definition, the bones, nerves, tendons, and vessels of his affected limbs were broken into three pieces, and the right thumb was nearly completely severed from middle segment of the first metacarpal bone (Figure 1). Preoperative X-ray of the right elbow joint was intact (Figure 2). The wounds were seriously contaminated and there was no blood supply. The boy had no additional injuries led our decision to replant the right arm.

The operation started $3 \mathrm{~h}$ after the accident and was carried out simultaneously by two replantation teams and three procedures under brachial plexus anesthesia (the right forearm team; wrist and hand team). The procedures of the replantation were fixation of bone, anastomoses of subcutaneous artery, veins and nerve; and closure of wound in turn. After blood flows of the severed limb were stopped with a pneumatic tourniquet, the severed limb replantation was performed by the first team; meanwhile, the severed thumb was replanted by the second team. Treated with a rapid debridement, the vessels, nerves and tendons of the 3 broken ends were all separated and tagged. The ends of the radius and ulna fixation was established with the plate and screw; wrist fused with cross kirschner wires; the fracture of the first metacarpal bone was fixed with cross kirschner wires after replacement. All of the flexor and extensor tendon ends of the forearm wrist and thumb were repaired with $0 / 1$ pull-out tendon sutures, and the stumps of muscles were sutured respectively. After successful anastomoses on radial and ulnar arteries and their branches of the forearm wrist and thumb, which successful to recover the blood supply, the 4 subcutaneous and accompanying veins of them were anastomosed as reflux via, and then the median, ulnaris and radialis nerves and their branches were sutured at different levels by $9 / 0$ and 10/0 microscopy. After losing the tourniquet, the injured had good blood supply. Then the wound was closed with silk sutures. The soft tissues and bones were repaired successfully; the replanted limb, hand and thumb were survived well. The total ischemia time was noted as $6 \mathrm{~h}$ and the duration of the replantation was $10 \mathrm{~h}$. Post-operatively the boy was safely admitted to ward.

Two and a half months later, there was a partial necrosis of the right wrist ministry but the forearm itself was intact. Intraoperative findings: $5 \times 3 \mathrm{~cm}$ skin necrosis of right ulnar palm, and skin resection combined with free skin grafting were performed. Postoperative effect of skin grafting was satisfied. After 3 months later, the replanted limb was well viability and the patient was able to perform simple activities (Figures 3 and 4 ).

\section{Discussion}

In the early 1960s, Millesi et al. [1] who did some experimental work outlined substantial improvements in the clinical results of peripheral nerve repair. Although modern microsurgery techniques have made advances after several decades, Lack of worth-while regeneration of nerves is a deterrent factor of replantation [2]. Furthermore, replantation surgeons need to consider the impact of replantation on each patient's quality of life, including the functional and esthetic [3] Our case was unusual in replantation of severed limb. We think that the vascular, nerve and tendon repair are very important in replantation of multi-segmental amputation because this could influence the success of replantation and recovery of function. The vascular injury can lead to thrombosis; thrombus formation rate of the multi-segmental amputation was very high, good vascular anastomosis, the applications of anticoagulant and antispasmodic were the key elements of success of the replantation. Nerve recovery is believed to be good because

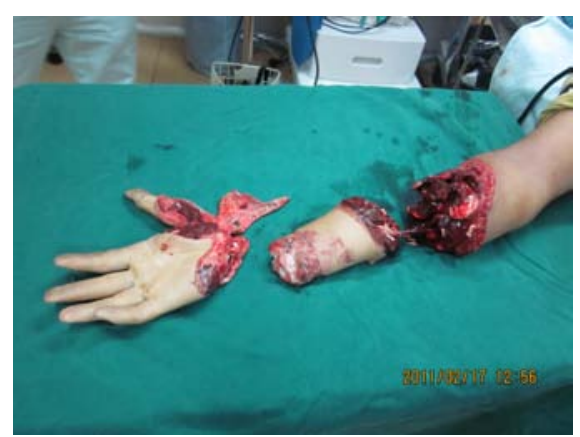

Figure 1: Compete amputation of the right thumb, the wrist and the forearm.

*Corresponding author: Jiangning Wang, Department of Plastic and Reconstructive Surgery, Luhe Hospital, Capital Medical University, 82 Xinhua South Road, Tongzhou, Beijing 101149, P.R. China, E-mail: yefeng541009@sina.com

Received March 12, 2013; Accepted April 27, 2013; Published May 06, 2013

Citation: Yin Y, Si X, Wang J, Jin C, Chao Z (2013) Replantation of a Multi-leve Upper Extremity Amputation. Surgery S13: 001. doi:10.4172/2161-1076.S13-001

Copyright: ( 2013 Yin Y, et al. This is an open-access article distributed under the terms of the Creative Commons Attribution License, which permits unrestricted use, distribution, and reproduction in any medium, provided the original author and source are credited. 


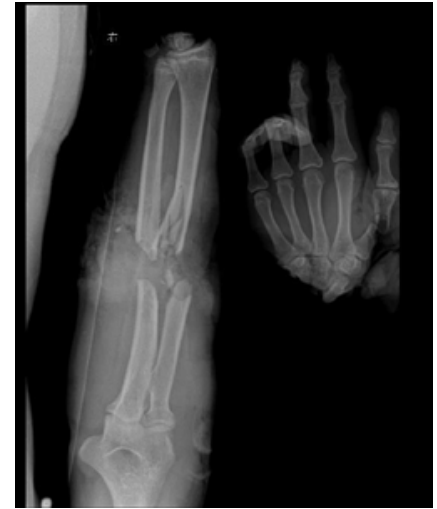

Figure 2: Preoperative X-ray of the right forearm.

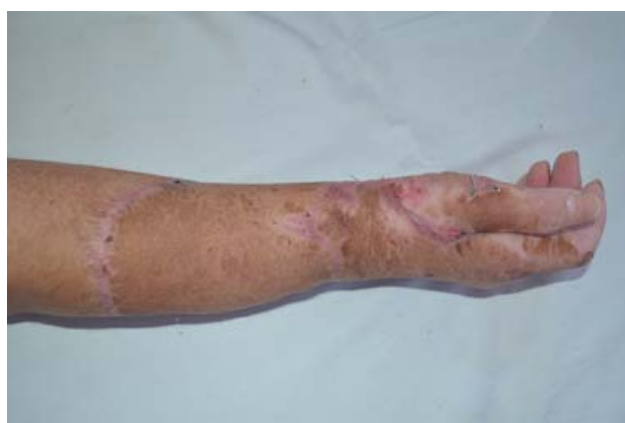

Figure 3: Postoperative X-ray of the newly replanted upper-limb after 3 months is shown.

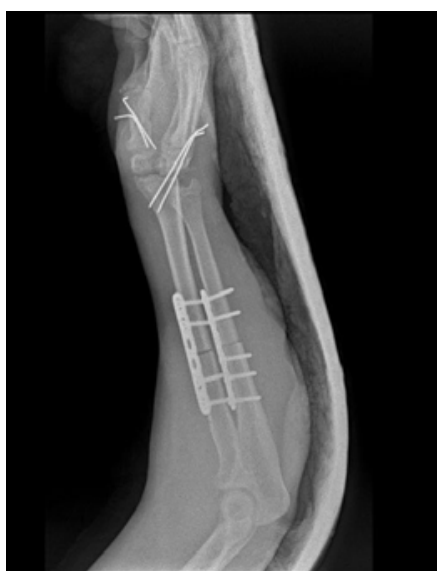

Figure 4: Postoperative view of the newly replanted upper-limb after 3 months is shown. of the phenomenon of the adjacent and spontaneous neurotization at the distance replantation. What's more, it may play a important role especially in younger patients [4]. Our case stated a replantation of upper extremity with associated a very rarely seen multi-level amputation type, the success of the replantation depends on the experienced surgeons' technology and functional training. The intact right elbow joint also helps the functional recovery of the patient [5].

\section{Conflicts of Interest}

We confirm that there are no known conflicts of interest associated with this publication and there has been no significant financial support for this work that could have influenced its outcome.

\section{Contributors}

All authors contributed to patient management; YF, YIN, and XL, SI wrote the report. We thank BC, XU, MR, Wang for helping to collect clinical images and data. Written consent to publish was obtained

\section{References}

1. Millesi H, Meissl G, Berger A (1976) Further experience with interfascicular grafting of the median, ulnar, and radial nerves. J Bone Joint Surg Am 58 209-218.

2. JAMES C WHITE (1968) Nerve Regeneration after Replantation of Severed Arms. Ann Surg 170: 715-719.

3. Kim WK, Lim JH, Han SK (1996) Fingertip replantations: clinical evaluation of 135 digits. Plast Reconstr Surg 98: 470-476.

4. Faivre S, Lim A, Dautel G, Duteille F, Merle M (2003) Adjacent and spontaneous neurotization after distal digital replantation in children. Plast Reconstr Surg 111: 159-165.

5. American Replantation Mission to China (1973) Replantation surgery surgery in China. Report of the American Replantation Mission to China. Plast Reconstr Surg 52: 476-489. 